\title{
镍催化格氏试剂与二芳基乙炔偶联反应制备四取代萗
}

\author{
陈锦杨 $^{a}$ 吴小波 ${ }^{b}$ 易荣楠 ${ }^{a}$ 许新华 $*, a$ \\ ( ${ }^{a}$ 湖南大学化学化工学院 长沙 410082) \\ ( 湖北省纤维检验局 武汉 420100)
}

\begin{abstract}
摘要 报道了一个新颖的方法来制备四取代菜化物, 该方法通过 $\mathrm{NiCl}_{2}$ 催化下的二芳基乙炔与格氏试剂的无配体偶联 反应实现. 该方法避免了已有方法中的特殊配体和昂贵催化剂的使用. 此外, 本方法还具有反应条件温和, 产率高, 底 物适用范围广等优点, 为多取代菜的制备提供了一个有效的方法.

关键词 镍催化偶联反应; 四取代萘; 格氏试剂; 二芳基乙炔
\end{abstract}

\section{Nickel-Catalyzed Coupling of Grignard Reagents and Diaryl Acetylenes for Synthesis of Tetra-substituted Naphthalenes}

\author{
Chen, Jinyang $^{a} \quad \mathrm{Wu}$, Xiaobo $^{b} \quad$ Yi, Rongnan $^{a} \quad \mathrm{Xu}$, Xinxua $^{*, a}$ \\ ( ${ }^{a}$ College of Chemistry and Chemical Engineering, Hunan University, Changsha 410082) \\ ( ${ }^{b}$ Fiber Inspection Bureau of Hubei Province, Wuhan 420100)
}

\begin{abstract}
A novel approach for the synthesis of tetra-substituted naphthalenes is demonstrated through the ligand-free coupling of a wide range of alkynes with Grignard reagents catalyzed by $\mathrm{NiCl}_{2}$, avoiding the use of special ligands and expensive catalysts used in previous methods. Other outstanding features include mild reaction conditions, good yields and wide functional group tolerance. The protocol provides an efficient method for the synthesis of highly substituted naphthalenes.

Keywords nickel-catalyzed coupling; tetra-substituted naphthalenes; Grignard reagents; diaryl acetylene
\end{abstract}

自从 1977 年 Heeger 课题组 ${ }^{[1]}$ 发现高导电聚乙炔以 来, 有机稠环共轭分子作为新一代的有机高分子功能材 料引起了广大科研工作者的兴趣 ${ }^{[2 \sim 9]}$. 与传统的无机固 体电子器件相比, 有机电子器件的性能可以通过分子设 计进行优化, 从而实现有机半导体的结构的多样化 ${ }^{[10]}$. 有机稠环化合物作为重要的中间体, 在甾体药物合成方 面也具有重要应用 ${ }^{[11]}$. 同时, 有机稠环共轭分子具有丰 富的物理化学性质, 它不仅可以作为二维石墨片段的分 子模型单元 ${ }^{[12]}$, 也可以作为一类极具发展潜质的光电 功能材料体系 ${ }^{[13 \sim 16]}$. 其在有机发光二极管 ${ }^{[17]}$ 和有机场 效应晶体管 ${ }^{[18]}$ 等有机材料方面具有重要的应用. 此外, 由于有机稠环共轭分子具有稳定性好、溶解性好及其良 好的苂光特性, 多取代芳环化合物在光电化学方面也具 有重要的应用 ${ }^{[19,20]}$.

近年来, 很多关于多取代菜化合物的合成方法被相
继报道 ${ }^{[21 ~ 23]}$. 过渡金属催化下的同系化反应是合成多

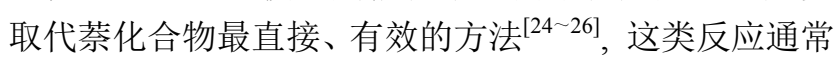
以芳基衍生物作为底物，与两分子炔烃化合物发生偶联 反应得到多取代菜化合物. 钯催化下芳基碘化物与二芳 基乙炔的偶联反应也是制备多取代荎化合物的重要方 法 ${ }^{[27]}$ ，该方法使用简单、易得的芳基碘化物和炔烃作为 反应底物，底物适用范围广. 但是该方法通常得到具有 多种多取代荎异构体的混合物. 由于这些异构体极性相 似, 因此难以进行分离、纯化. 2008 年, Cheng 课题组 ${ }^{[28]}$ 以 $\mathrm{Ni}$ (dppe) $\mathrm{Br}_{2}$ 为催化剂, 催化多取代二碘代芳基化物 与炔烃反应制备多取代萗，该方法对不同的二碘代芳基 化物和内炔具有很好的适用性. 但该方法中使用的难以 制备的多取代二碘代芳基化物作为反应底物. Miura 等 ${ }^{[29]}$ 报道了 $\mathrm{Rh}$ 催化下, 芳基硼酸与二芳基乙炔反应制 备多取代荎化物，该方法使用昂贵的铑催化剂，反应在

\footnotetext{
* Corresponding author. E-mail: xhx1581@hnu.edu.cn

Received December 24, 2016; revised February 21, 2017; published online March 14, 2017.

Project supported by the Hunan Provincal Science and Technology Major Projects (No. 2014FJ1010).

湖南省科技重大专项基金(No. 2014FJ1010)资助项目.
} 
高温下才能有效进行. 其他使用 $\mathrm{Rh}^{[30,31]} 、 \mathrm{Ir}^{[32]} 、 \mathrm{Pd}^{[33,34]}$ 或 $\mathrm{Fe}^{[35]}$ 作为催化剂制备多取代萗的方法也相继被报道, 这些反应通常需要使用昂贵的催化剂, 配体或特殊的试 剂作为底物. Nakamura 等 ${ }^{[36]}$ 报道了在 $\mathrm{Fe}(\mathrm{acac})_{3} / 1,10$-菲 罗啉催化体系下，二芳基乙炔与格氏试剂反应制备多取 代菜, 该反应只有在昂贵的 1,10-菲罗啉的条件下才能 有效进行. 有些方法使用难以制备的试剂作为反应底 物, 增加了反应的操作难度. 因此, 急需开发出一种操 作简便、反应条件温和的方法来制备该类多取代䒺化合 物.

本文以廉价的 $\mathrm{NiCl}_{2}$ 作为催化剂, 通过向内炔和 $\mathrm{NiCl}_{2}$ 的反应体系中逐滴滴加格氏试剂的方法, 较高产 率地制备一系列多取代菜化合物. 该方法具有操作简 便, 反应条件温和, 产率高等优点, 为多取代荎化合物 的制备提供了一个方便、有效的方法.

\section{1 结果与讨论}

在氮气保护下，向二苯乙炔 $(2.0 \mathrm{mmol}) 、 \mathrm{NiCl}_{2}(0.25$ $\mathrm{mmol})$ 和四氢呋喃(THF) $(2.0 \mathrm{~mL})$ 的混合液中加入苯基 溴化镁 $(1.0 \mathrm{mmol})$, 反应在室温条件下进行 $15 \mathrm{~h}$, 反应 获得 $23 \%$ 的产率. 当向反应体系中加入一倍量的 $\mathrm{Ag}_{2} \mathrm{CO}_{3}$ 时, 反应产率增加到 $42 \% . \mathrm{Ag}_{2} \mathrm{CO}_{3}$ 在反应过程 中可能起到氧化剂的作用, 从而促进反应有效进行. 然 后, 我们以二苯乙炔和苯基溴化镁的反应作为模型反应 (Scheme 1), 系统考察不同催化剂、反应温度以及格氏试 剂的添加方式对反应的影响，结果见表 1 .

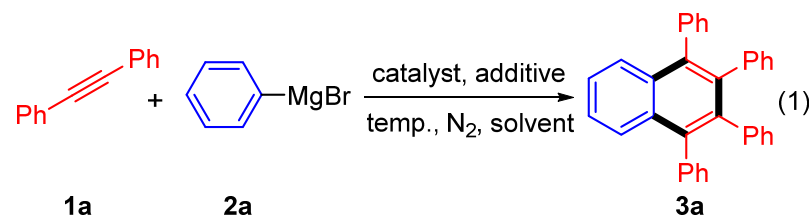

由表 1 可知, 以无水 $\mathrm{NiCl}_{2}$ 作为催化剂时, 二苯乙炔 与苯基溴化镁的反应获得最高的产率. 当以 $\mathrm{NiCl}_{2} \cdot 6 \mathrm{H}_{2} \mathrm{O}$ 作为催化剂时, $\mathrm{NiCl}_{2} \cdot 6 \mathrm{H}_{2} \mathrm{O}$ 中的结晶水使格氏试剂部分 水解, 导致反应产率降低. 格氏试剂的加入方式对反应 影响很大, 当格氏试剂一次性加入到反应体系中时, 反 应产率均较低. 而通过逐滴滴加的方式, 反应产率明显 增加(Table1, Entry 9). 主要因为在 $\mathrm{NiCl}_{2}$ 存在下, 过量 的格氏试剂容易发生自身偶联反应生成联苯. 反应在室 温 $\left(25{ }^{\circ} \mathrm{C}\right)$ 下有效进行, 升高或降低反应温度, 反应产率 均有所下降. 反应温度的升高有助于格氏试剂自身偶联 反应的进行. 因此以无水 $\mathrm{NiCl}_{2}$ 作为反应的最佳催化剂, 反应温度为 $25{ }^{\circ} \mathrm{C}$, 采用逐滴滴加格氏试剂的方法作为 最佳的反应方法.

随后以无水 $\mathrm{NiCl}_{2}$ 作为催化剂, 氮气氛围下, 以二
表 1 不同催化剂及反应温度对反应的影响 ${ }^{a}$

Table 1 Influence of various catalyst and temperature to the reaction

\begin{tabular}{clcc}
\hline Entry & \multicolumn{1}{c}{ Catalyst } & $t{ }^{\circ} \mathrm{C}$ & Yield $^{b} / \%$ of 3a \\
\hline 1 & $\mathrm{NiCl}_{2}$ & 25 & 42 \\
2 & $\mathrm{NiCl}_{2} \cdot 6 \mathrm{H}_{2} \mathrm{O}$ & 25 & 8 \\
3 & $\mathrm{NiF}_{2}$ & 25 & 20 \\
4 & $\mathrm{Ni}(\mathrm{OAc})_{2}$ & 25 & 15 \\
5 & $\left.\mathrm{Ni}_{(\mathrm{acac}}\right)_{2}$ & 25 & 14 \\
6 & $\mathrm{NiBr}_{2}$ & 25 & 28 \\
7 & $\mathrm{Ni}_{2}\left(\mathrm{PPh}_{3}\right)_{2} \mathrm{Cl}_{2}$ & 25 & 31 \\
8 & $\mathrm{Ni}_{2}(\mathrm{dppe}) \mathrm{Cl}_{2}$ & 25 & 26 \\
$9^{c}$ & $\mathrm{NiCl}_{2}$ & 25 & 75 \\
$10^{c}$ & $\mathrm{NiCl}_{2}$ & 0 & 38 \\
$11^{c}$ & $\mathrm{NiCl}_{2}$ & 50 & 41 \\
\hline
\end{tabular}

${ }^{a}$ Reaction conditions: dibenzenyl $(2.0 \mathrm{mmol})$, catalyst $(25 \mathrm{~mol} \%)$, THF (2.0 $\mathrm{mL}), \mathrm{Ag}_{2} \mathrm{CO}_{3}(1.0 \mathrm{mmol})$, under nitrogen atmosphere, phenyl magnesium bromide $(1.0 \mathrm{mmol})$ was added in one portion. ${ }^{b} \mathrm{GC}$ yields. ${ }^{c}$ phenyl magnesium bromide was added dropwise to the reaction mixture under nitrogen atmosphere.

苯乙炔 $(2.0 \mathrm{mmol})$ 与苯基溴化镁 $(1.0 \mathrm{mmol})$ 的反应作为 模型反应，考察催化剂用量以及反应时间对反应的影 响, 结果见表 2 .

表 2 催化剂用量及时间对反应的影响 ${ }^{a}$

Table 2 Influence of catalyst amount and reaction time to the reaction

\begin{tabular}{cccc}
\hline Entry & $\mathrm{NiCl}_{2} / \mathrm{mol}^{\circ} \%$ & $t / \mathrm{h}$ & $\mathrm{Yield}^{b} \% \%$ \\
\hline 1 & 100 & 15 & 81 \\
2 & 50 & 15 & 80 \\
3 & 25 & 15 & 73 \\
4 & 20 & 15 & 68 \\
5 & 10 & 15 & 42 \\
6 & 5 & 15 & 18 \\
7 & 20 & 20 & 75 \\
8 & 20 & 25 & 82 \\
9 & 20 & 30 & 82 \\
\hline
\end{tabular}

${ }^{a}$ Reaction conditions: dibenzenyl $(2.0 \mathrm{mmol})$, phenyl magnesium bromide (1.0 mmol), $\mathrm{NiCl}_{2}$, THF $(2.0 \mathrm{~mL}), 25{ }^{\circ} \mathrm{C}, \mathrm{Ag}_{2} \mathrm{CO}_{3}(1.0 \mathrm{mmol})$, under nitrogen atmosphere. ${ }^{b} \mathrm{GC}$ yields.

由表 2 可知, 催化剂用量的增加可以缩短反应进行 的时间，当反应时间延长至 $25 \mathrm{~h}$ 时， $20 \mathrm{~mol} \%$ 的催化剂 便可以催化反应有效进行, 反应获得 $82 \%$ 的产率. 因此 选择 $20 \mathrm{~mol} \%$ 的催化剂用量作为反应的最佳用量.

在以上基础上, 我们继续以二苯乙炔与苯基溴化镁 的反应作为模型反应, 在 $20 \mathrm{~mol} \%$ 催化剂的催化下, 反 应在氮气保护下进行 $25 \mathrm{~h}$, 考察溶剂对反应的影响, 结 果见表 3.

由表 3 可知, 不同的溶剂对反应具有一定的影响, 当使用甲苯、乙醚或四氢呋喃作为反应溶剂时, 反应效 果较好. 以 $\mathrm{THF}$ 作为反应溶剂时, 该反应获得最高的产 率, 反应产率为 $82 \%$, 因此选择 THF 作为最佳反应溶 
剂.

表 3 溶剂对反应的影响 ${ }^{a}$

Table 3 Influence of solvent to the reaction

\begin{tabular}{clc}
\hline Entry & Solvent & Yield $^{b} \%$ \\
\hline 1 & $\mathrm{CH}_{3} \mathrm{CN}$ & 72 \\
2 & Ether & 69 \\
3 & $\mathrm{CH}_{2} \mathrm{Cl}_{2}$ & 52 \\
4 & $\mathrm{DMF}$ & 67 \\
5 & Toluene & 76 \\
6 & 1,4-Dioxane & 65 \\
7 & THF & 82 \\
\hline
\end{tabular}

${ }^{a}$ Reaction conditions: dibenzenyl (2.0 mmol), phenyl magnesium bromide (1.0 $\mathrm{mmol}), \mathrm{NiCl}_{2}(20 \mathrm{~mol} \%)$, solvent $(2.0 \mathrm{~mL}), 25{ }^{\circ} \mathrm{C}, \mathrm{Ag}_{2} \mathrm{CO}_{3}(1.0 \mathrm{mmol})$, under nitrogen atmosphere. ${ }^{b} \mathrm{GC}$ yields.

继而, 以二苯乙炔与苯基溴化镁的反应作为模型反 应, 在 $20 \mathrm{~mol} \%$ 催化剂的催化下, 以 THF 作为反应溶剂, 反应在氮气保护下进行 $25 \mathrm{~h}$, 考察不同配比苯乙炔与苯 基溴化美条件下的反应情况，结果见表 4.

表 4 不同配比苯乙炔与苯基溴化镁条件下的反应情况 ${ }^{a}$

Table 4 Influence of the various ratio of dibenzenyl and phenyl magnesium bromide to the reaction

\begin{tabular}{ccc}
\hline Entry & $\mathbf{1 a}: \mathbf{2 a}$ & Yield $^{b} / \%$ \\
\hline 1 & $2: 1$ & 82 \\
2 & $3: 1$ & 67 \\
3 & $1: 1$ & 53 \\
4 & $1: 2$ & 38 \\
5 & $1: 3$ & 25 \\
\hline
\end{tabular}

${ }^{a}$ Reaction conditions: dibenzenyl, phenyl magnesium bromide, $\mathrm{NiCl}_{2}(20$ $\mathrm{mol} \%)$, THF $(2.0 \mathrm{~mL}), 25{ }^{\circ} \mathrm{C}, \mathrm{Ag}_{2} \mathrm{CO}_{3}(1.0 \mathrm{mmol})$, under nitrogen atmosphere. ${ }^{b} \mathrm{GC}$ yields.

由表 4 可知，当二苯乙炔与苯基溴化镁的投料比为 $2: 1$ 时, 反应获得最高的产率, 随着格氏试剂量的增 加, 反应生成的副产物增加, 因此选择二苯乙炔与苯基 澳化镁的投料比为 $2: 1$ 作为该反应的最佳投料比.

最后, 以二苯乙炔与苯基溴化美的反应作为模型反 应, 在 $20 \mathrm{~mol} \%$ 催化剂的催化下, 以 THF 作为反应溶剂, 反应在氮气保护下进行 $25 \mathrm{~h}$, 苯乙炔与苯基溴化镁的投 料比为 $2: 1$, 考察不同添加剂对反应的影响结果见表 5 .

由表 5 可知, 在无添加剂下, 反应产率较低(Table 5, Entry 1). 向反应体系中加入一当量的碱, 并不能使反应 产率得到明显增加(Table 5, Entries 2, 3). 而在反应体系 中加入 $\mathrm{Ag}_{2} \mathrm{CO}_{3}$ 或 $\mathrm{Cu}(\mathrm{OAc})_{2}$ 时, 反应产率均得到明显增 加 (Table 5, Entries 4,5). 主要是因为 $\mathrm{Ag}_{2} \mathrm{CO}_{3}$ 或 $\mathrm{Cu}(\mathrm{OAc})_{2}$ 在反应过程中起到了氧化剂的作用, 从而促 进反应有效进行, 反应产率增加. $50 \mathrm{~mol} \%$ 的 $\mathrm{Ag}_{2} \mathrm{CO}_{3}$ 就 能使反应有效进行, 反应获得 $82 \%$ 的产率, 因此选择 $\mathrm{Ag}_{2} \mathrm{CO}_{3}(50 \mathrm{~mol} \%$ )作为最佳添加剂.

在上述实验基础上, 系统考察各种二芳基乙炔与格 氏试剂的反应情况(Eq. 2), 结果见表 6.
表 5 不同添加剂对反应的影响 ${ }^{a}$

Table 5 Influence of the various additive to the reaction

\begin{tabular}{clc}
\hline Entry & Additive $(\mathrm{mol} \%)$ & Yield $^{b} \%$ \\
\hline 1 & None & 40 \\
2 & $\mathrm{Na}_{2} \mathrm{CO}_{3}(100)$ & 41 \\
3 & $\mathrm{~K}_{2} \mathrm{CO}_{3}(100)$ & 46 \\
4 & $\mathrm{Ag}_{2} \mathrm{CO}_{3}(100)$ & 82 \\
5 & $\mathrm{Cu}(\mathrm{OAc})_{2}(100)$ & 65 \\
6 & $\mathrm{Ag}_{2} \mathrm{CO}_{3}(50)$ & 82 \\
7 & $\mathrm{Ag}_{2} \mathrm{CO}_{3}(25)$ & 69 \\
\hline
\end{tabular}

${ }^{a}$ Reaction conditions: dibenzenyl (2.0 mmol), phenyl magnesium bromide (1.0 $\mathrm{mmol}), \mathrm{NiCl}_{2}(20 \mathrm{~mol} \%)$, THF $(2.0 \mathrm{~mL}), 25{ }^{\circ} \mathrm{C}$, under nitrogen atmosphere. ${ }^{b} \mathrm{GC}$ yields.

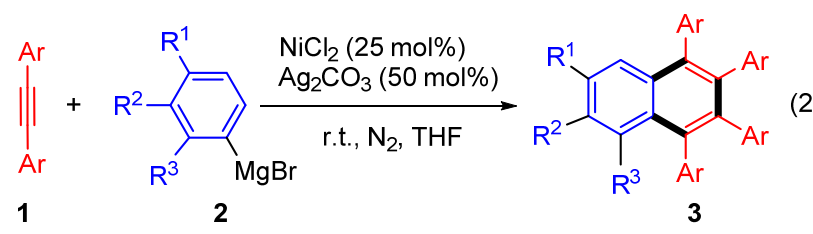

表 6 不同二芳基乙炔与格氏试剂的反应产率 ${ }^{a}$

Table 6 Yields of reaction of diaryl acetylene with Grignard reagents

\begin{tabular}{cccclcc}
\hline Entry & $\mathrm{R}^{1}$ & $\mathrm{R}^{2}$ & $\mathrm{R}^{3}$ & \multicolumn{1}{c}{$\mathrm{Ar}$} & Product & Yield $^{b} \%$ \\
\hline 1 & $\mathrm{H}$ & $\mathrm{H}$ & $\mathrm{Me}$ & $\mathrm{Ph}$ & $\mathbf{3 b}$ & 80 \\
2 & $\mathrm{H}$ & $\mathrm{Me}$ & $\mathrm{H}$ & $\mathrm{Ph}$ & $\mathbf{3 c}$ & 78 \\
3 & $\mathrm{Me}$ & $\mathrm{H}$ & $\mathrm{H}$ & $\mathrm{Ph}$ & $\mathbf{3 c}$ & 82 \\
4 & $\mathrm{OMe}$ & $\mathrm{H}$ & $\mathrm{H}$ & $\mathrm{Ph}$ & $\mathbf{3 d}$ & 75 \\
5 & $\mathrm{H}$ & $\mathrm{H}$ & $\mathrm{H}$ & $p-\mathrm{CH}_{3} \mathrm{C}_{6} \mathrm{H}_{4}$ & $\mathbf{3 e}$ & 70 \\
6 & $\mathrm{H}$ & $\mathrm{H}$ & $\mathrm{H}$ & $p-\mathrm{CH}_{3} \mathrm{OC}_{6} \mathrm{H}_{4}$ & $\mathbf{3 f}$ & 68 \\
7 & $\mathrm{Me}$ & $\mathrm{H}$ & $\mathrm{H}$ & $p-\mathrm{CH}_{3} \mathrm{C}_{6} \mathrm{H}_{4}$ & $\mathbf{3 g}$ & 76 \\
8 & $\mathrm{H}$ & $\mathrm{Me}$ & $\mathrm{H}$ & $p-\mathrm{CH}_{3} \mathrm{C}_{6} \mathrm{H}_{4}$ & $\mathbf{3 g}$ & 75 \\
9 & $\mathrm{H}$ & $\mathrm{H}$ & $\mathrm{OMe} \mathrm{Ph}$ & $\mathbf{3 h}$ & 65 \\
10 & $\mathrm{H}$ & $\mathrm{H}$ & $\mathrm{OMe}$ & $p-\mathrm{CH}_{3} \mathrm{C}_{6} \mathrm{H}_{4}$ & $\mathbf{3 i}$ & 65 \\
$11^{c}$ & $\mathrm{H}$ & $\mathrm{H}$ & $\mathrm{H}$ & $p-\mathrm{ClC}_{6} \mathrm{H}_{4}$ & & Trace \\
12 & $\mathrm{Et}$ & $\mathrm{H}$ & $\mathrm{H}$ & $p-\mathrm{CF}_{3} \mathrm{C}_{6} \mathrm{H}_{4}$ & $\mathbf{3 j}$ & 84 \\
\hline${ }^{a} \mathrm{R}$ & &
\end{tabular}

${ }^{a}$ Reaction conditions: diaryl acetylene $(2.0 \mathrm{mmol})$, Grignard reagents $(1.0$ mmol), $\mathrm{NiCl}_{2}(20 \mathrm{~mol} \%)$, THF $(2.0 \mathrm{~mL}), 25{ }^{\circ} \mathrm{C}, \mathrm{Ag}_{2} \mathrm{CO}_{3}(0.5 \mathrm{mmol})$, under nitrogen atmosphere. ${ }^{b}$ Isolated yields. ${ }^{c} \mathrm{GC}$ yields.

由表 6 可知，该反应对不同取代的格氏试剂和二芳 基乙炔均具有一定的适用性，反应均获得较高的产率. 芳基溴化镁苯环对位上的给电子基 $(\mathrm{OMe}, \mathrm{Me})$ 对反应影 响不大(Table 6, Entries 2 6). 但给电子基 $(\mathrm{OMe})$ 的位置 对反应具有一定的影响，当芳基溴化镁的邻位被甲氧基 取代时，反应产率较低(Table 6, Entries 9, 10), 可能是因 为空间位阻的影响. 而当芳基溴化镁的苯环上的取代基 为 $\mathrm{Cl}$ 时，反应几乎得不到预期的目标产物，反应管中有 大量絮状物生成(Table 6, Entry 11), 且大部分二苯基乙 炔可以通过柱层析得到回收. 可能是因为在 $\mathrm{NiCl}_{2}$ 存在 下，氯代苯基格氏试剂发生自身偶联反应，生成分子量 较大的聚合物. 二芳基乙炔上的取代基对反应具有一定 的影响，当二芳基乙炔的苯环上的取代基为给电子基 
(OMe, Me) 时反应产率有所下降(Table 6, Entries 5 8). 而当二芳基乙炔苯环上的取代基为强吸电子基 $\left(\mathrm{CF}_{3}\right)$ 时, 反应获得较高的产率 $(\mathbf{3} \mathbf{j}, 84 \%)$. 可能是因为 $\mathrm{CF}_{3}$ 的强吸 电子作用增加了二芳基乙炔的反应活性, 从而使得反应 有效进行.

\section{2 结论}

与已有方法相比, 本方法使用廉价的 $\mathrm{NiCl}_{2}$ 作为催 化剂, 催化二芳基乙炔与格式试剂反应, 较高产率地获 得了一系列的四取代荎化合物. 该反应具有条件温和, 易于操作, 原料价格低廉, 收率高等优点, 避免了特殊 配体以及昂贵催化剂的使用, 为多取代萗的合成提供了 一个简便、有效的方法.

\section{3 实验部分}

\section{1 仪器与试剂}

${ }^{1} \mathrm{H}$ NMR (TMS 为内标), ${ }^{13} \mathrm{C}$ NMR(以 TMS 为内标) 用 INOVA-400 型仪测定, 质谱由 HP5989A 测定, 硅胶 为青岛海洋化工厂产品. 溶剂四氢呋喃(THF)、乙腈 $\left(\mathrm{CH}_{3} \mathrm{CN}\right)$ 、乙醚 $\left(\mathrm{Et}_{2} \mathrm{O}\right)$ 、二氯甲烷 $\left(\mathrm{CH}_{2} \mathrm{Cl}_{2}\right)$ 、甲苯(Toluene) 和 1,4-二氧六环 (1,4-dioxane)通过标准方法进行无水处 理，原料二芳基乙炔根据文献[37]方法制备，格氏试剂 均从阿拉丁试剂公司购买, 苯基溴化镁 $(2.5 \mathrm{~mol} / \mathrm{L}$ in $\mathrm{THF}$ ), 对 4-甲苯基溴化镁(1.0 mol/L in THF), 3-甲苯基 溴化镁 $(1.0 \mathrm{~mol} / \mathrm{L}$ in THF), 2-苯基溴化镁 $(1.0 \mathrm{~mol} / \mathrm{L}$ in THF), 4-甲氧苯基溴化镁(1.0 mol/L in THF), 2-甲氧苯基 澳化镁 $(1.0 \mathrm{~mol} / \mathrm{L}$ in THF $)$.

\section{2 实验方法}

室温下, 氮气保护下, 将苯基澳化镁 (2a) (1.0 $\mathrm{mmol}$ )逐滴滴加到二苯基乙炔(1a) $(2.0 \mathrm{mmol}), \mathrm{NiCl}_{2}$ (0.25 mmol), $\mathrm{Ag}_{2} \mathrm{CO}_{3}(0.5 \mathrm{mmol}$ 和 $2.0 \mathrm{~mL}$ 干燥的 THF 的反应液中. 反应混合液在室温、氮气保护下反应 $25 \mathrm{~h}$. 反应结束后, 向反应液中加入 $2.0 \mathrm{~mL}$ 甲醇淬灭反应, 并 用乙酸乙酯萃取 $(10 \mathrm{~mL} \times 3)$, 合并有机相, 并用无水 $\mathrm{Na}_{2} \mathrm{SO}_{4}$ 干燥. 去除溶剂, 经柱层析得 1,2,3,4-四苯基萗 (3a) ${ }^{[21]}$. ${ }^{1} \mathrm{H}$ NMR $\left(\mathrm{CDCl}_{3}, 400 \mathrm{MHz}\right) \delta: 7.65 \sim 7.63(\mathrm{~m}$, $2 \mathrm{H}), 7.40 \sim 7.37(\mathrm{~m}, 2 \mathrm{H}), 7.26 \sim 7.22(\mathrm{~m}, 9 \mathrm{H}), 7.05 \sim 7.04$ (m, 2H,), $6.84(\mathrm{~m}, 9 \mathrm{H}) ;{ }^{13} \mathrm{C}$ NMR $\left(\mathrm{CDCl}_{3}, 100 \mathrm{MHz}\right) \delta$ : $140.54,139.59,138.89,138.42,132.03,131.13,127.53$, $127.49,126.99,126.43,125.88,125.33$; MS (EI) $m / z$ : 432.2 .

5-甲基-1,2,3,4-四苯基萗 $(\mathbf{3 b})^{[21]}$ : ${ }^{1} \mathrm{H} \mathrm{NMR}\left(\mathrm{CDCl}_{3}\right.$, $400 \mathrm{MHz}) \delta: 7.52(\mathrm{~d}, J=8.4 \mathrm{~Hz}, 1 \mathrm{H}), 7.26 \sim 7.10(\mathrm{~m}$, $13 \mathrm{H}), 6.81 \sim 6.75(\mathrm{~m}, 9 \mathrm{H}), 1.95(\mathrm{~s}, 3 \mathrm{H}) ;{ }^{13} \mathrm{C} \mathrm{NMR}\left(\mathrm{CDCl}_{3}\right.$, $100 \mathrm{MHz}) \delta: 142.94,140.75,140.68,140.46,140.34$,
$139.08,138.49,138.07,135.94,133.40,131.64,131.30$, $131.12,130.92,130.34,127.91,127.49,126.83,126.48$, $126.34,126.28,126.19,125.46,125.23,125.03,25.37$; MS (EI) $m / z: 446.2$.

6-甲基-1,2,3,4-四苯基萗 $(3 \mathbf{c})^{[21]}$ : ${ }^{1} \mathrm{H}$ NMR $\left(\mathrm{CDCl}_{3}\right.$, $400 \mathrm{MHz}) \delta: 7.54(\mathrm{~d}, J=8.8 \mathrm{~Hz}, 1 \mathrm{H}), 7.40(\mathrm{~s}, 1 \mathrm{H}), 7.21 \sim$ $7.17(\mathrm{~m}, 11 \mathrm{H}), 6.83(\mathrm{~s}, 10 \mathrm{H}), 2.37(\mathrm{~s}, 3 \mathrm{H}) ;{ }^{13} \mathrm{C} \mathrm{NMR}$ $\left(\mathrm{CDCl}_{3}, 100 \mathrm{MHz}\right) \delta: 140.74,140.68,139.79,139.01$, $137.27,138.04,137.82,135.67,132.20,131.43,131.40$, $131.38,131.35,130.31,128.17,127.95,127.92,127.56$, $127.55,126.96,126.59,126.41,126.39,125.91,125.30$, 21.91; MS (EI) $m / z: 446.2$.

6-甲氧基-1,2,3,4-四苯基萗(3d) ${ }^{[21]}:{ }^{1} \mathrm{H} \mathrm{NMR}\left(\mathrm{CDCl}_{3}\right.$, $400 \mathrm{MHz}) \delta: 7.56(\mathrm{~d}, J=9.2 \mathrm{~Hz}, 1 \mathrm{H}), 7.24 \sim 7.17(\mathrm{~m}$, $10 \mathrm{H}), 7.06(\mathrm{~d}, J=9.2 \mathrm{~Hz}, 1 \mathrm{H}), 6.95(\mathrm{~s}, 1 \mathrm{H}), 6.84(\mathrm{~m}, 10 \mathrm{H})$, $3.68(\mathrm{~s}, 3 \mathrm{H}) ;{ }^{13} \mathrm{C} \mathrm{NMR}\left(\mathrm{CDCl}_{3}, 100 \mathrm{MHz}\right) \delta: 157.55$, $140.73,140.59,139.77,139.72,139.42,138.35,137.29$, $136.79,133.28,131.48,131.28,131.27,131.22,128.72$, $127.51,126.54,126.40,125.38,125.28,125.21,118.02$, 105.66, 55.16; MS (EI) $m / z: 462.2$.

1,2,3,4-四(4-甲苯基)芸(3e $)^{[21]}:{ }^{1} \mathrm{H} \mathrm{NMR}\left(\mathrm{CDCl}_{3}, 400\right.$ MHz) $\delta: 7.62 \sim 7.59(\mathrm{~m}, 2 \mathrm{H}), 7.35 \sim 7.33(\mathrm{~m}, 2 \mathrm{H}), 7.08 \sim$ $7.03(\mathrm{~m}, 8 \mathrm{H}), 6.71 \sim 6.65(\mathrm{~m}, 8 \mathrm{H}), 2.32(\mathrm{~s}, 6 \mathrm{H}), 2.10(\mathrm{~s}$, $6 \mathrm{H}) ;{ }^{13} \mathrm{C} \mathrm{NMR}\left(\mathrm{CDCl}_{3}, 100 \mathrm{MHz}\right) \delta: 139.10,138.29$, 137.74, 136.79, 135.61, 134.31, 132.19, 131.14, 131.12, $128.20,127.24,126.97,125.52,21.25,21.09$; MS (EI) $m / z$ : 488.2.

1,2,3,4-四(4-甲氧苯基)萘 $(3 \mathbf{f f})^{[21]}:{ }^{1} \mathrm{H} \mathrm{NMR}\left(\mathrm{CDCl}_{3}\right.$, $400 \mathrm{MHz}) \delta: 7.65 \sim 7.63(\mathrm{~m}, 1 \mathrm{H}), 7.37 \sim 7.35(\mathrm{~m}, 1 \mathrm{H})$, $7.10(\mathrm{~d}, J=8.0 \mathrm{~Hz}, 2 \mathrm{H}), 6.79(\mathrm{~d}, J=8.4 \mathrm{~Hz}, 2 \mathrm{H}), 6.71(\mathrm{~d}$, $J=8.4 \mathrm{~Hz}, 2 \mathrm{H}), 6.43$ (d, $J=8.0 \mathrm{~Hz}, 2 \mathrm{H}), 3.80$ (s, 3H), 3.63 $(\mathrm{s}, 3 \mathrm{H}) ;{ }^{13} \mathrm{C}$ NMR $\left(\mathrm{CDCl}_{3}, 100 \mathrm{MHz}\right) \delta: 157.89,156.87$, 139.13, 138.16, 133.36, 132.37, 132.30, 132.27, 132.16, 126.96, 125.60, 113.02, 112.15, 55.14, 54.91; MS (EI) $\mathrm{m} / \mathrm{z}$ : 552.2 .

6- 甲基-1,2,3,4-四 (4- 甲苯基) 萗 (3g): ${ }^{1} \mathrm{H} \quad \mathrm{NMR}$ $\left(\mathrm{CDCl}_{3}, 400 \mathrm{MHz}\right) \delta: 7.50(\mathrm{~d}, J=8.4 \mathrm{~Hz}, 1 \mathrm{H}), 7.37(\mathrm{~s}$, $1 \mathrm{H}), 7.17(\mathrm{~d}, J=7.6 \mathrm{~Hz}, 1 \mathrm{H}), 7.07 \sim 7.02(\mathrm{~m}, 8 \mathrm{H}), 6.70 \sim$ $6.63(\mathrm{~m}, 8 \mathrm{H}), 2.36(\mathrm{~s}, 3 \mathrm{H}), 2.31(\mathrm{~s}, 3 \mathrm{H}), 2.30(\mathrm{~s}, 3 \mathrm{H}), 2.08$ $(\mathrm{s}, 6 \mathrm{H}) ;{ }^{13} \mathrm{C}$ NMR $\left(\mathrm{CDCl}_{3}, 100 \mathrm{MHz}\right) \delta: 139.19,138.21$, $138.10,137.92,137.85,137.65,136.97,136.92,135.51$, $135.46,135.16,134.19,132.32,131.19,131.15,130.44$, $128.17,128.15,127.74,127.19,126.90,125.85,21.78$, 21.26, 21.24, 21.06; MS (EI) $m / z: 502.3$.

5-甲氧基-1,2,3,4-四苯基萗 $(3 \mathbf{h})^{[38]}$ : ${ }^{1} \mathrm{H} \mathrm{NMR}\left(\mathrm{CDCl}_{3}\right.$, 
$400 \mathrm{MHz}) \delta: 7.25 \sim 7.18(\mathrm{~m}, 7 \mathrm{H}), 7.09(\mathrm{~s}, 4 \mathrm{H}), 6.84 \sim 6.78$ $(\mathrm{m}, 12 \mathrm{H}), 3.37(\mathrm{~s}, 3 \mathrm{H}) ;{ }^{13} \mathrm{C} \mathrm{NMR}\left(\mathrm{CDCl}_{3}, 100 \mathrm{MHz}\right) \delta$ : $157.21,143.87,140.63,140.55,140.14,138.18,136.63$, $134.08,131.49$, 131.30, 131.25, 131.12, 129.84, 127.53, $127.47,126.57,126.46,126.33,126.22,126.02,125.22$, $125.00,124.78,123.73,120.11,106.91,55.81$; MS (EI) $m / z: 462.2$.

5- 甲氧基-1,2,3,4-四(4-甲苯基)菜 (3i): ${ }^{1} \mathrm{H}$ NMR $\left(\mathrm{CDCl}_{3}, 400 \mathrm{MHz}\right) \delta: 7.04 \sim 7.02(\mathrm{~m}, 2 \mathrm{H}), 6.98 \sim 6.95(\mathrm{~m}$, $5 \mathrm{H}), 6.94 \sim 6.93(\mathrm{~m}, 3 \mathrm{H}), 6.88 \sim 6.81(\mathrm{~m}, 2 \mathrm{H}), 6.78 \sim 6.65$ $(\mathrm{m}, 2 \mathrm{H}), 6.63 \sim 6.52(\mathrm{~m}, 5 \mathrm{H}), 3.36(\mathrm{~s}, 3 \mathrm{H}), 2.30(\mathrm{~s}, 3 \mathrm{H})$, $2.25(\mathrm{~s}, 3 \mathrm{H}), 2.07(\mathrm{~s}, 6 \mathrm{H}) ;{ }^{13} \mathrm{C} \mathrm{NMR}\left(\mathrm{CDCl}_{3}, 100 \mathrm{MHz}\right) \delta$ : $140.20,139.64,138.08,137.37,136.53,135.52,134.11$, $133.86,133.69$, 131.27, 131.09, 130.92, 130.73, 129.67, $129.50,128.63,128.57,128.14,127.49,127.12,126.88$, $126.65,126.01,125.59,120.22,107.01,55.99,21.41$, 21.25, 21.20, 21.07; MS (EI) $m / z: 518.3$.

6-乙基-1,2,3,4-四(4-三氟甲基苯基)菜(3j): ${ }^{1} \mathrm{H}$ NMR $\left(\mathrm{CDCl}_{3}, 400 \mathrm{MHz}\right) \delta: 7.56 \sim 7.48(\mathrm{~m}, 5 \mathrm{H}), 7.38 \sim 7.32(\mathrm{~m}$, $6 \mathrm{H}), 7.17$ (d, $J=7.6 \mathrm{~Hz}, 4 \mathrm{H}), 6.93$ (d, $J=7.6 \mathrm{~Hz}, 4 \mathrm{H})$, $2.72 \sim 2.70(\mathrm{~m}, 2 \mathrm{H}), 1.21(\mathrm{t}, J=7.6 \mathrm{~Hz}, 3 \mathrm{H}) ;{ }^{13} \mathrm{C} \mathrm{NMR}$ $\left(\mathrm{CDCl}_{3}, 100 \mathrm{MHz}\right) \delta: 143.56,143.27,142.64,137.69$, $137.37,137.02$, 136.11, 131.99, 131.40, 131.36, 131.31, $130.29,128.53,128.24,126.84,125.19,124.91,124.53$, 124.10, 122.48, 29.16, 15.67; MS (ESI) m/z: 732.2.

辅助材料(Supporting Information) 所有目标产物的 ${ }^{1} \mathrm{H}$ NMR 和 ${ }^{13} \mathrm{C}$ NMR 谱. 这些材料可以免费从本刊网站 (http://sioc-Journal.cn/)上下载.

\section{References}

[1] Chiang, C. K.; Fincher, C. R.; Park, Y. W.; Heeger, A. J.; Shirakawa, H.; Louis, E. J.; Gau, S. C.; MacDiarmid, A. G. Phys. Rev. Lett. 1977, 39, 1098

[2] Anthony, J. E. Angew. Chem., Int. Ed. 2008, 47, 452.

[3] Wu, W.; Liu, Y.; Zhu, D. Chem. Soc. Rev. 2010, 39, 1489.

[4] Zhang, J. L.; Xiao, C.; Zhai, L. J.; Wang, X. J.; Bi, F. Q.; Wang, B. Z. Chin. J. Org. Chem. 2016, 36, 1197 (in Chinese). (张俊林, 肖川, 翟连杰, 王锡杰, 毕福强, 王伯周, 有机化学, 2016, 36, 1197.)

[5] Wu, J.; Kong, H. H.; Ding, M. W. Chin. J. Org. Chem. 2016, 36, 1662 (in Chinese)

(武静, 孔晗晗, 丁明武, 有机化学, 2016, 36, 1662.)

[6] Yang, R.; Cai, X. D.; Ding, L. M. Acta Chim. Sinica 2015, 73, 281 (in Chinese).
(杨瑞，蔡雪入，丁黎明，化学学报, 2015, 73, 281.)

[7] Ge, T.; Song, J.; yYao, Z. G.; Xu, F. Chin. J. Org. Chem. 2015, 35, 1570 (in Chinese). (葛恬, 宋洁, 姚志刚, 徐凡, 有机化学, 2015, 35, 1570.)

[8] Gong, X.; Xie, X.; Chen, N.; Zheng, C.; Zhu, J.; Chen, R.; Huang, W.; Gao, D. Chin. J. Chem. 2015, 33, 967.

[9] Wang, L.; Wang, L.; Huang, J.; Yu, G. Chin. J. Chem. 2015, 33, 948.

[10] Dimitrakopoulos, C. D.; Malenfant, P. R. L. Adv. Mater. 2002, 14, 99.

[11] Narasimhan, N. S.; Bapat, C. P. J. Chem. Soc., Perkin Trans. I 1984, 1435.

[12] Wang, C.; Dong, H.; Hu, W.; Liu, Y.; Zhu, D. Chem. Rev. 2012, $112,2208$.

[13] Murphy, A. R.; Frechet, J. M. J. Chem. Rev. 2007, 107, 1066.

[14] Burroughes, J. H.; Bradley, D. D. C.; Brown, A. R.; Marks, R. N.; Mackay, K.; Friend, R. H.; Burns, P. L.; Holmes, A. B. Nature 1990, 347, 539 .

[15] Coakley, K. M.; McGehee, M. D. Chem. Mater. 2004, 16, 4533

[16] Someya, T.; Katz, H. E.; Gelperin, A.; Lovinger, A. J.; Dodabalapur, A. Appl. Phys. Lett. 2002, 81, 3079.

[17] Katz, H. E.; Bao, Z. J. Phys. Chem. B 2000, 104, 671.

[18] Bendikov, M.; Wudl, F.; Perepichka, D. F. Chem. Rev. 2004, 104, 4891.

[19] Kaur, I.; Stein, N. N.; Kopreski, R. P.; Miller, G. P. J. Am. Chem. Soc 2009, 131, 3424.

[20] Ahmed, E.; Briseno, A. L.; Xia, Y.; Jenekhe, S. A. J. Am. Chem. Soc. 2008, 130, 1118.

[21] Fukutani, T.; Hirano, K.; Satoh, T.; Miura, M. J. Org. Chem. 2011, $76,2867$.

[22] Takahashi. T.; Li, Y.; Stepnicja, P.; Kitamura, M.; Liu, Y.; Nakajima, K.; Kotora, M. J. Am. Chem. Soc. 2002, 124, 576

[23] Umeda, N.; Tsurugi, H.; Satoh, T.; Miera, M. Angew. Chem., Int. Ed. 2008, 47, 4019.

[24] Takahashi, T.; Kitamura, M.; Shen, B.; Nakajima, K. J. Am. Chem. Soc. 2000, 122, 12876.

[25] Bennett, M. A.; Hockless, C. R.; Wenger, E. Organometallics 1995, 14, 2091.

[26] Bowles, D. M.; Anthony, J. E. Org. Lett. 2000, $2,85$.

[27] Kawasaki, S.; Satoh, T.; Miura, M.; Nomura, M. J. Org. Chem. 2003, 68, 6836.

[28] Hsieh, J-C.; Cheng, C-H. Chem. Commun. 2008, 2992.

[29] Fukutani, T.; Hirano, K.; Satoh, T.; Miura, M. Org. Lett. 2009, 11, 5198.

[30] Uto, T.; Shimazu, M.; Ueura, K.; Tsurugi, H.; Satoh, T.; Miura, M. J. Org. Chem. 2008, 73, 298.

[31] Geng, K.; Fan, Z.; Zhang, A. Org. Chem. Front. 2016, 3, 349.

[32] Ueura, K.; Satoh, T.; Miura, M. J. Org. Chem. 2007, 72, 5362.

[33] Jafarpour, F.; Hazrati, H.; Nouraldinmousa, S. Org. Lett. 2013, 15, 3816.

[34] Cant, A. A.; Roberts, L.; Greaney, M. F. Chem. Commun. 2010, 46, 8671.

[35] Adak, L.; Yoshikai, N. Tetrahedron 2012, 68, 5167.

[36] Ilies, L.; Matsumoto, A.; Kobayashi, M.; Yoshikai, N.; Nakamura, E. Synlett 2012, 23, 2381.

[37] Thakur, K. G.; Sekar, G. Synthesis 2009, 16, 2785.

[38] Wu, Y.-T.; Huang, K.-H.; Shin, C.-C.; Wu, T.-C. Chem.-Eur. J. 2008, 14, 6697. 